\title{
Evaluation of disposable trap column nanoLC-FAIMS-MS/MS for the proteomic analysis of FFPE tissue
}

Stephan Eckert ${ }^{l, 2,3, * \text {; Yun-Chien Chang }}{ }^{l, *}$; Florian P. Bayer ${ }^{l}$; Matthew The ${ }^{l}$; Peer-Hendrik Kuhn ${ }^{2,3}$; Wilko Weichert ${ }^{2,3}$; Bernhard Kuster ${ }^{1,3,4 *}$

\#: These authors contributed equally

${ }^{1}$ Chair of Proteomics and Bioanalytics, Technical University of Munich (TUM), 85354 Freising, Germany

${ }^{2}$ Institute of Pathology, Technical University of Munich (TUM), 81675 Munich, Germany

${ }^{3}$ German Cancer Consortium (DKTK), Partner-site Munich and German Cancer Research Center (DKFZ), 69120 Heidelberg, Germany

${ }^{4}$ Bavarian Biomolecular Mass Spectrometry Center (BayBioMS), Technical University of Munich (TUM), 85354 Freising, Germany

* Corresponding author: Bernhard Kuster (kuster@tum.de)

Tel: +49 8161715696, Fax: +49 8161715931

Correspondence to: kuster@tum.de

\section{Table of Content}

Figure S 1 - Influence of selected sample preparation parameters on the number of proteins identified from FFPE material.

Figure S 2 - Comparison of base peak chromatograms for differen amounts of peptides loaded

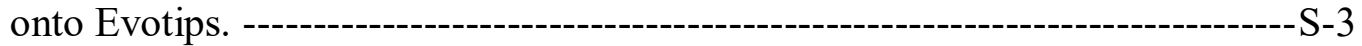

Figure S 3 - Characterization of FAIMS parameters. --------------------------S-5

Figure S 4 - Comparison of peptide and protein intensities without and with FAIMS when using different numbers of internal CVs. ----------------

Figure S 5 - Comparison of the number of unique peptides per protein without and with FAIMS when using different numbers of internal CVs. ------------------------------S-8

Figure S 6 - Comparison of different fractionation approaches. ---------------------------S-9

Figure S 7 - Number of peptides identified from three exemplary human FFPE tissues. ---S-10 

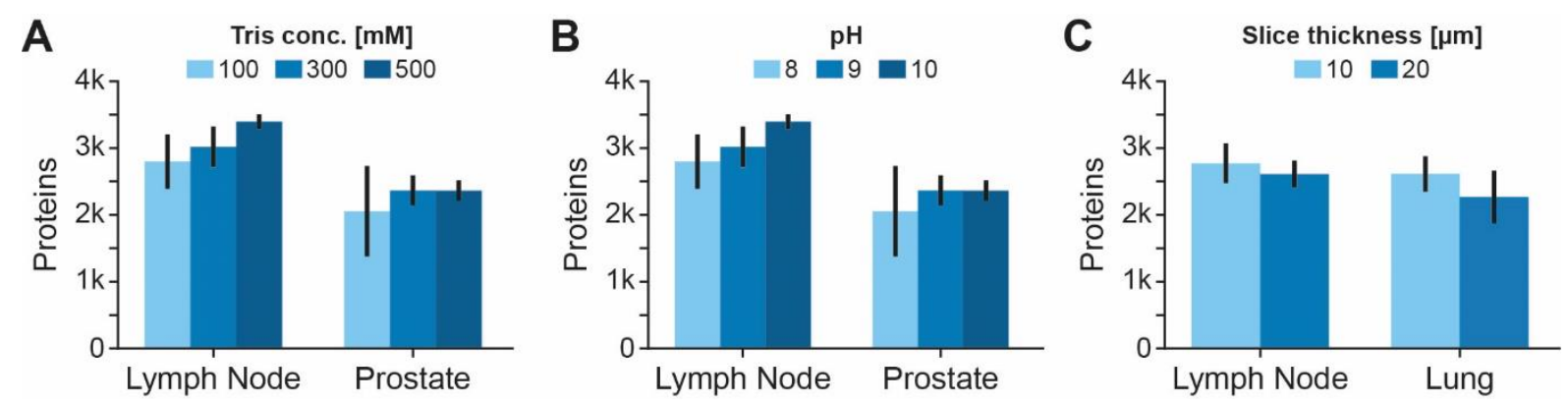

Figure S 1 - Influence of selected sample preparation parameters on the number of proteins identified from FFPE material. (A) Ionic strength of the extraction buffer, (B) $\mathrm{pH}$ of the extraction buffer and (C) thickness of tissue slices. For each analysis, $50 \mathrm{ng}$ of peptides digest were analysed by a $60 \mathrm{~min}$ LC-MS/MS run. Error bars indicate the standard error of mean (SEM). For the final extraction procedure, $500 \mathrm{mM}$ Tris, $\mathrm{pH} 9$ and a slice thickness of $10 \mu \mathrm{m}$ was chosen. 


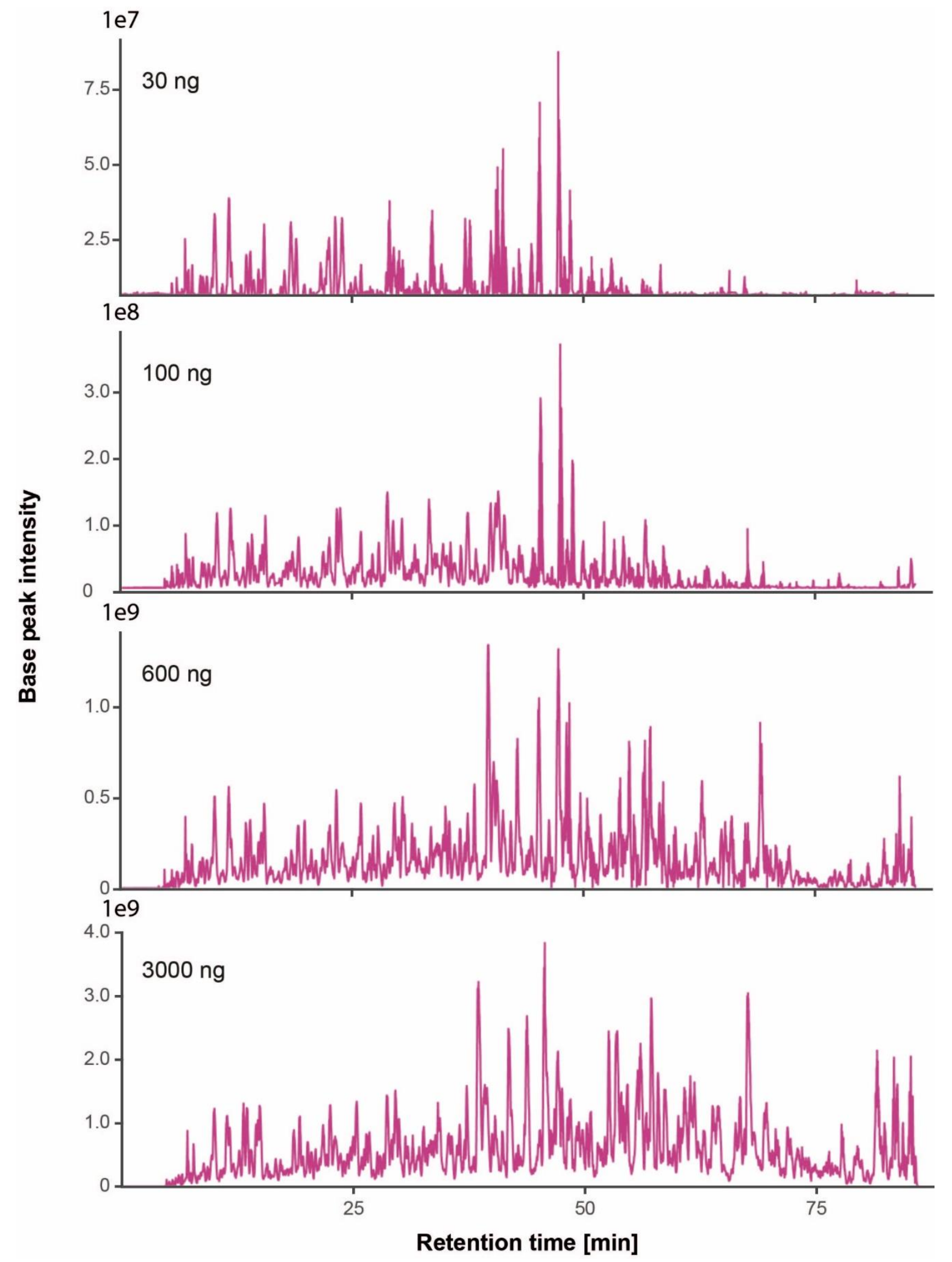

Figure S 2 - Comparison of base peak chromatograms across the LC retention time for different amounts of peptides (HeLa cell line digests) loaded onto Evotips (88 min gradient data shown). 
At low peptide loading amounts (100 ng and less), as substantial absolute loss (i.e. on the Evotips) of hydrophobic peptides was visible as base peak intensity was particular lower in the range from 60-88 min. In this area not only quantitative but qualitative changes were visible. Thus, the workflow presented here may have to be adjusted (e. g. using smaller bed volumes of the disposable trap column or direct injection onto a conventional nanoLC system) for smaller input amounts. 

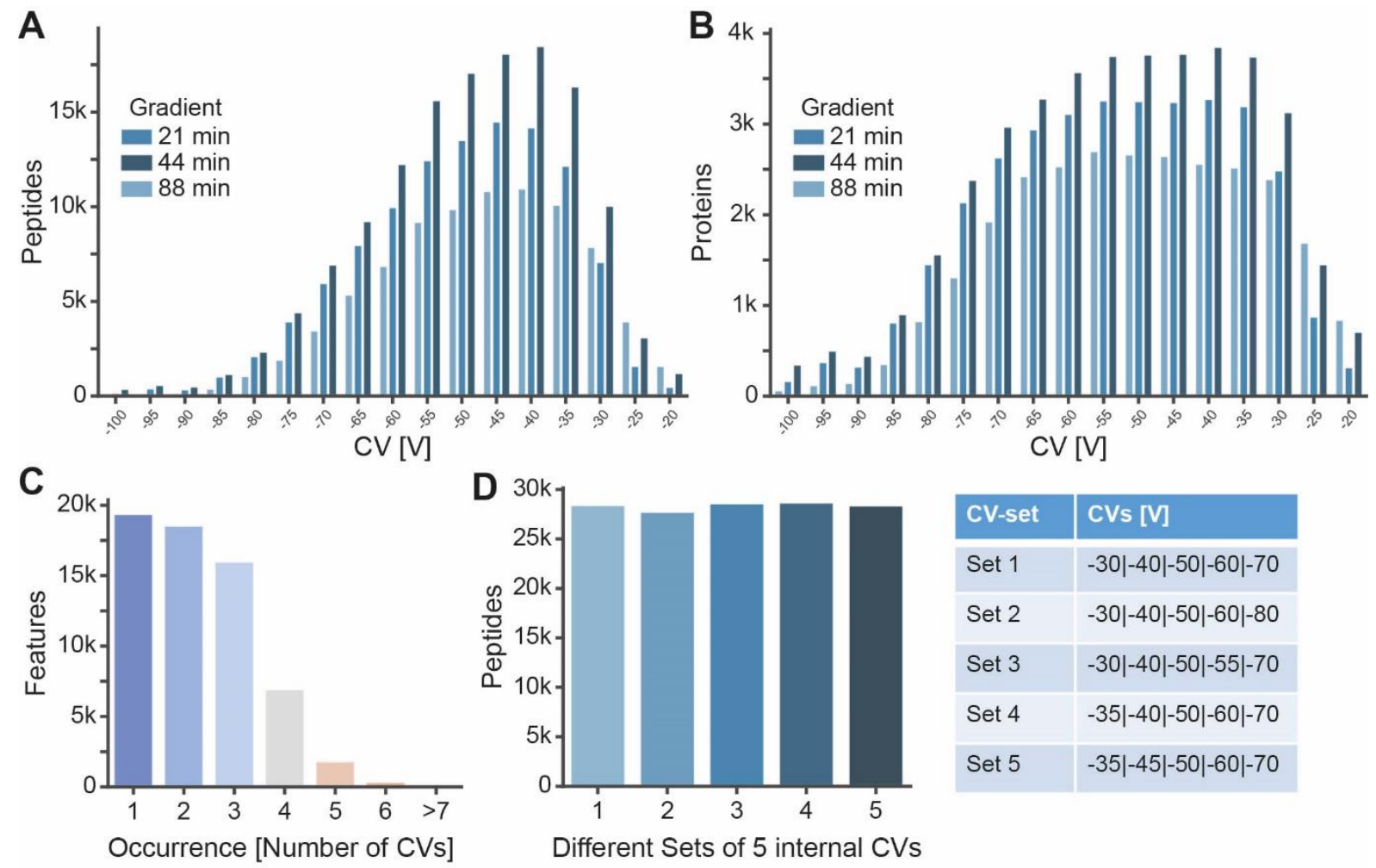

\begin{tabular}{|l|l|}
\hline CV-set & CVs [V] \\
\hline Set 1 & $-30 \mid-4$ 이-50|-60|-70 \\
\hline Set 2 & $-30|-40|-50|-60|-80$ \\
\hline Set 3 & $-30 \mid-40$ 이-50|-55|-70 \\
\hline Set 4 & $-35 \mid-40$ 이-50|-60|-70 \\
\hline Set 5 & $-35|-45|-50|-60|-70$ \\
\hline
\end{tabular}

E
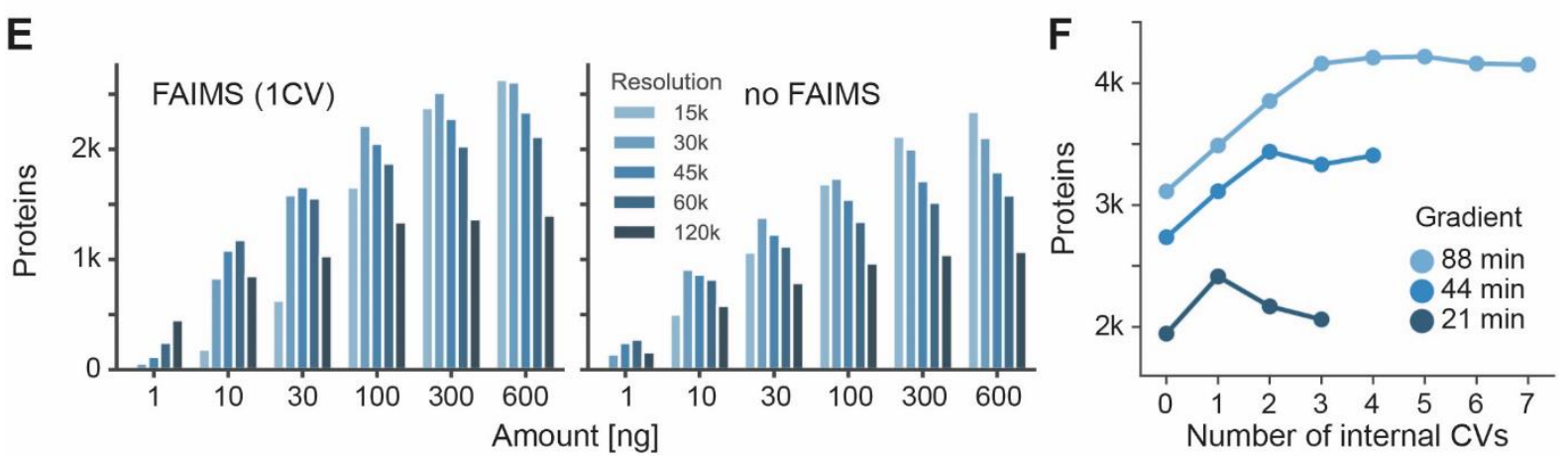

Figure S 3 - Characterization of FAIMS parameters. Number of(A) identified peptides and (B) proteins over a compensation voltage $(\mathrm{CV})$ ramp from $-20 \mathrm{~V}$ to $-100 \mathrm{~V}$ for gradient lengths of 21, 44, and 88 min. (C) Occurrence of peptide features (distinguishing charge states, modified peptide sequences etc.) at different CVs (88 min gradient data shown, $n=63,297)$. (D) Number of actually identified peptides applying five best combinations of five CVs ( 88 min gradient data shown). The table shows the CVs used in each set. (E) Number of identified proteins at different input amounts with different MS2 resolutions and matched maximum injection times (maxIT), with $(1 \mathrm{CV} ;-45 \mathrm{~V})$ and without FAIMS. At high peptide loading amounts, the fastest evaluated method (15k MS2 resolution, $22 \mathrm{~ms}$ maxIT) was the best choice. 
When decreasing the sample amount, a higher MS2 resolution with higher maxIT would be preferable. For FAIMS this trend was stronger, owing to the loss of intensity which was always observed when using only one CV. (F) Number of actually identified proteins when combining several CVs within an LC-MS/MS run of different gradient lengths. 

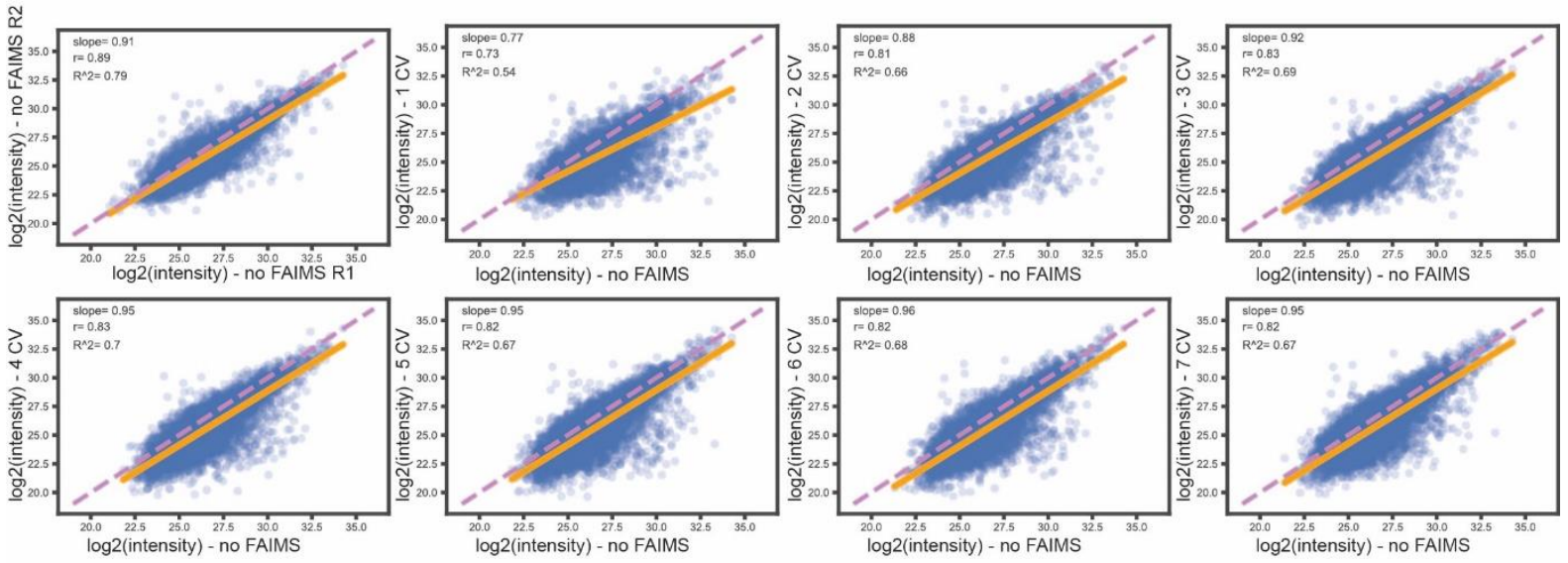

B
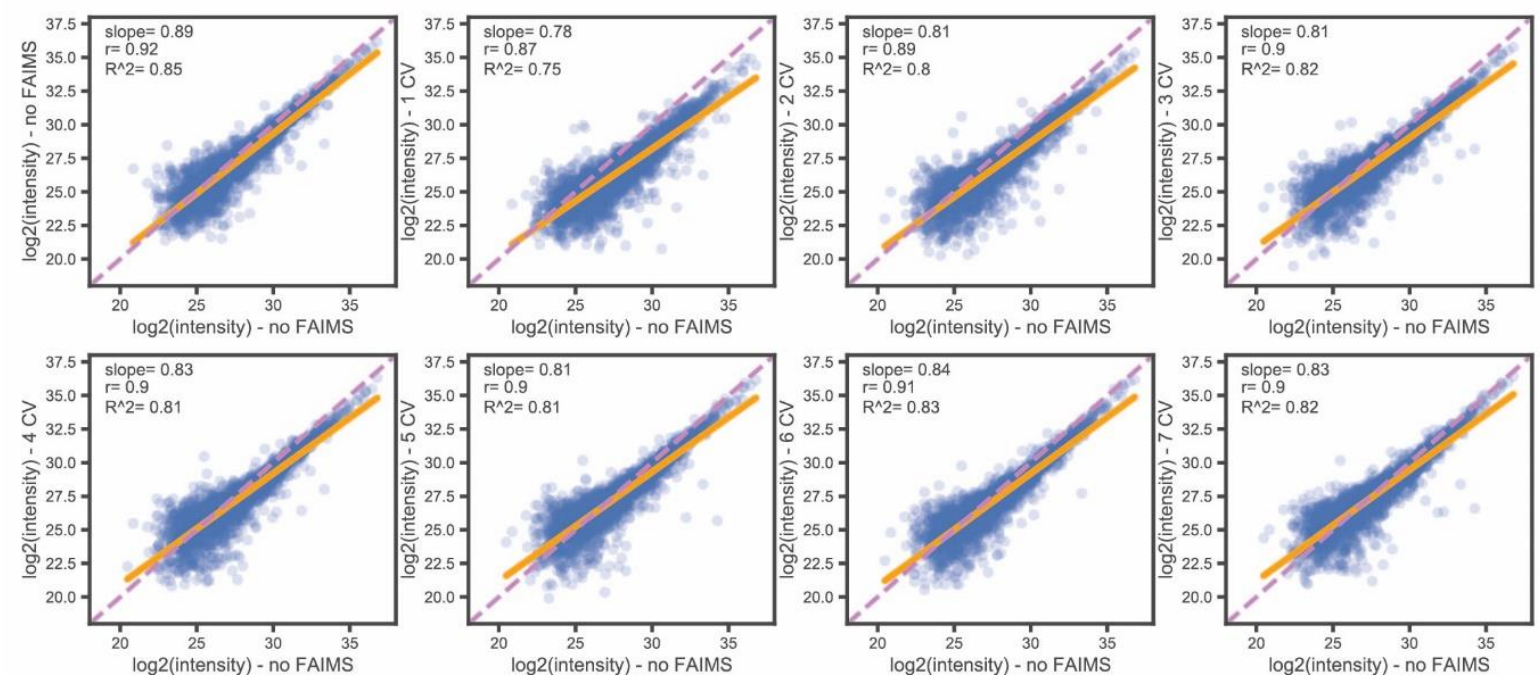

Figure S 4 - Comparison of peptide and protein intensities with and without FAIMS.

Scatterplots of (A) peptide precursor intensities ( $\log 2)$ and (B) protein intensities $(\log 2)$ when combining several CVs (0-7; where 0 represents a second replicate run without FAIMS) within a LC-MS/MS run compared to the intensities of the run without FAIMS. The dashed line marks the diagonal and the orange line depicts the linear regression ( $\mathrm{r}=$ Pearson correlation coefficient). Slope and R-squared value of linear regression line is shown. The correlation between peptide or protein intensities of nLC-MS/MS runs with and without FAIMS became better when more CVs were used. Thus, this suggests, that for the analysis on peptide and protein level at least 3 and 2 internal CVs (for an 88 min gradient) should be used, respectively. 

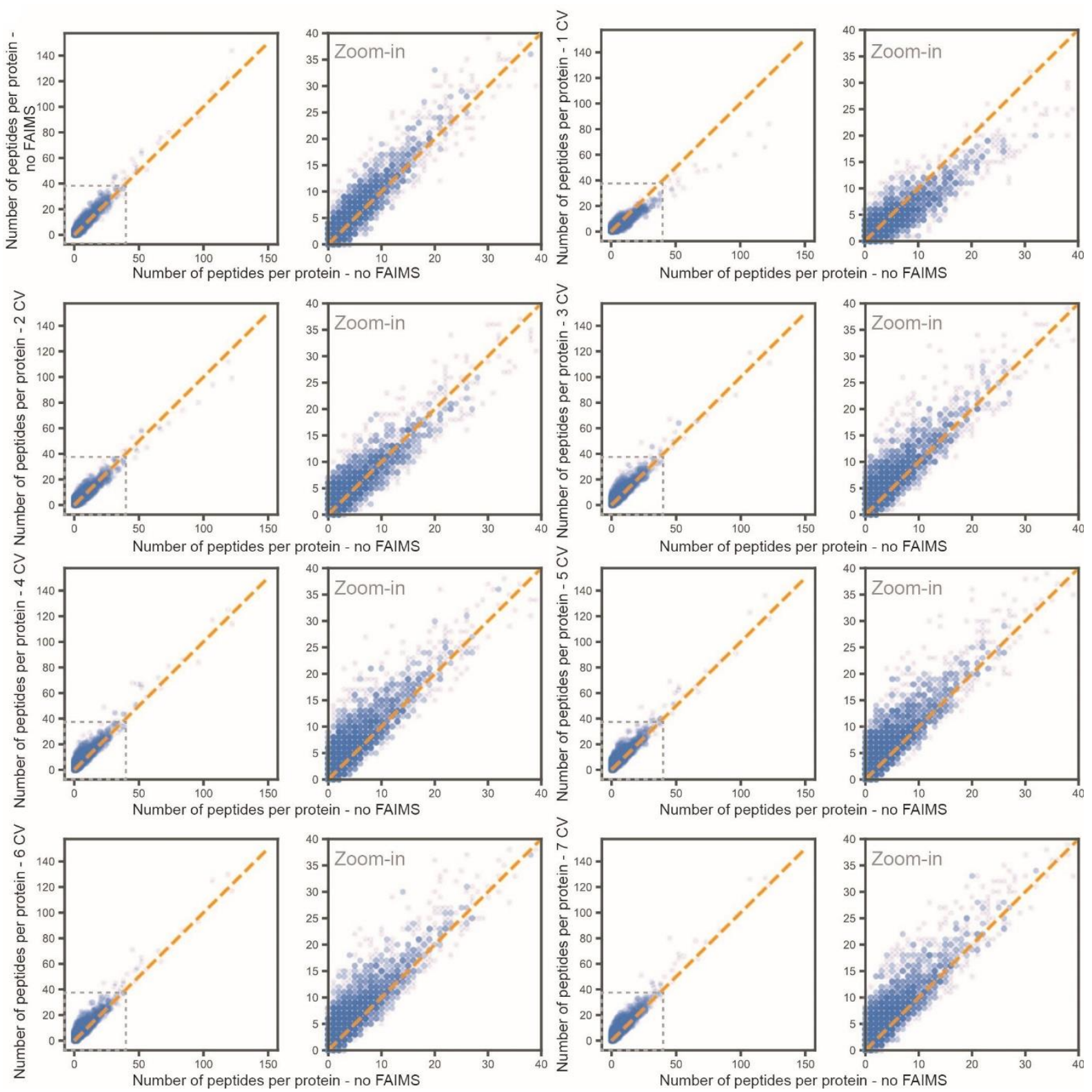

Figure S 5 - Comparison of the number of unique peptides per protein when combining several CVs (0-7; where 0 represents a second replicate run without FAIMS) within a nLC-MS/MS run compared to the run without FAIMS. The area marked with dashed line was zoomed-in and shown as the right plot labeled with "Zoom-in". The dashed line marks the diagonal. When using only one $\mathrm{CV}$, the number of peptides per protein is on average decreased compared to the run without FAIMS. This changed when more CVs were used, the number of peptides per protein increases and surpasses the numbers for the run without FAIMS. 
A

\begin{tabular}{|c|c|c|c|c|}
\hline Off-line bRP & - & - & + & + \\
\hline FAIMS & - & + & - & + \\
\hline Run1/Fraction1CVs [V] & - & -30 이 -4 이 -5 이-60|-70 & - & $-40 \mid-55$ \\
\hline Run2/Fraction2CVs [V] & & $-35|-45|-55|-65|-75$ & - & $-40 \mid-55$ \\
\hline Run3/Fraction3CVs [V] & & & - & $-40 \mid-55$ \\
\hline Run4/Fraction4CVs [V] & & & - & $-40 \mid-55$ \\
\hline
\end{tabular}

B
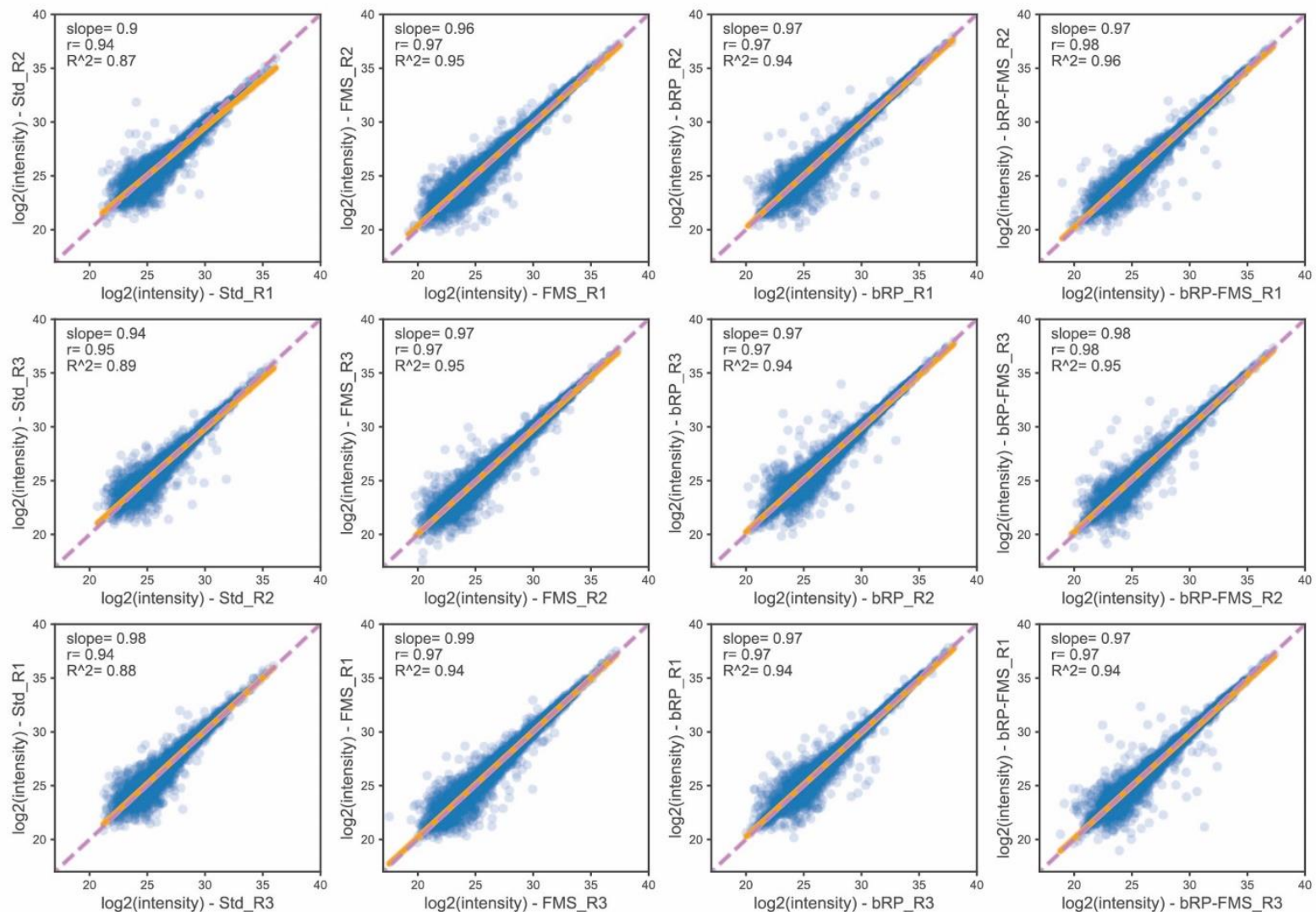

Figure S 6 - Comparison of proteomic depth obtained by FAIMS or off-line fractionation. (A) CVs used in the FAIMS and off-line bRP fractionation comparison. For the runs neither offline fractionated nor using FAIMS, two runs of 88 min gradient were shown ( $\mathrm{n}=3$ each). For the off-line bRP runs, 4 runs of 44 min gradient data were shown ( $\mathrm{n}=3$ each). (B) Scatterplots of $\log 2$ peptide precursor intensity of replicates of each fractionation method. The dashed line marks the diagonal and the orange line depicts the linear regression ( $\mathrm{r}=$ Pearson correlation coefficient). Slope and R-squared value of linear regression line was shown. 


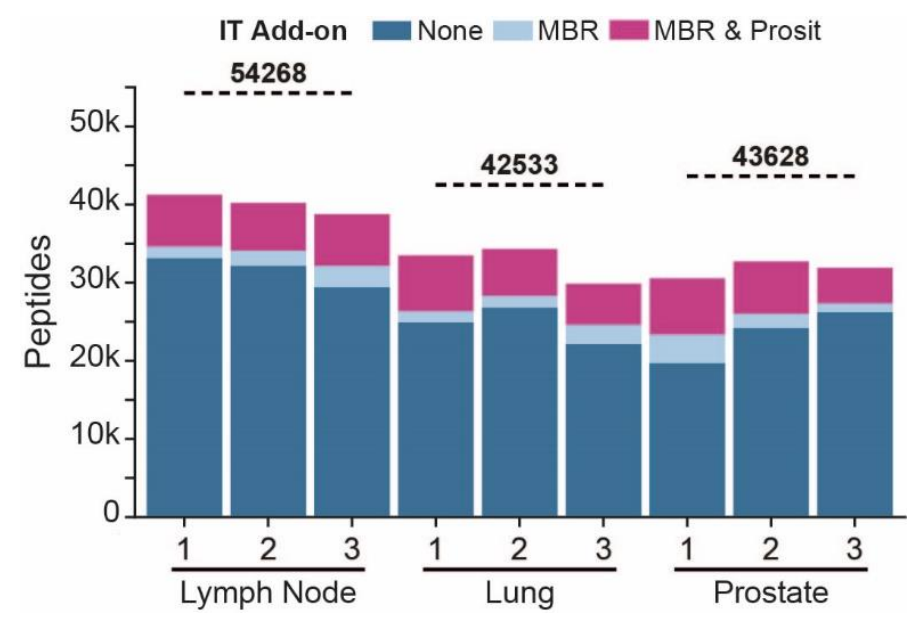

Figure S 7 - Number of peptides identified from three exemplary human FFPE tissues, lymph node, lung and prostate $(\mathrm{n}=3$ each) by standard database searching, match-between-runs enabled and re-scored by Prosit. Horizontal lines depict the total number of peptides identified in each tissue. 